\title{
Gaining an edge in the gut
}

Two articles, published in Nature and Environmental Microbiology, highlight the use of specific compounds in the intestine by two different pathogens to outcompete the native flora.

Salmonella enterica subsp. enterica serovar Typhimurium causes inflammation in the intestine, and Winter and colleagues now show that the bacterium utilizes the tetrathionate that is produced as a result of this host response. Tetrathionate has been used for nearly 90 years to enrich Salmonella spp. in cultures, as this bacterium, unlike many others, can use this compound as a terminal electron acceptor. Normally no tetrathionate is present in the gut. However, thiosulphate, which is produced in the intestines from the hydrogen sulphide secreted by the gut microbiota, can be converted

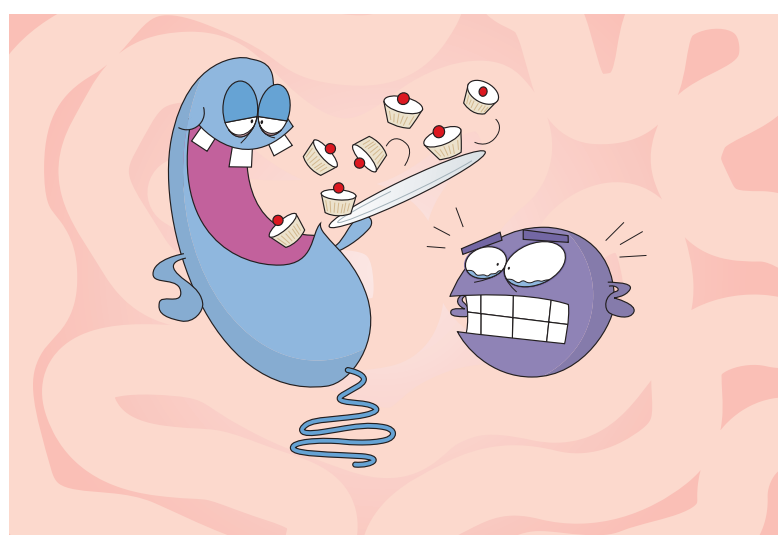

to tetrathionate by a strong oxidizing agent. As neutrophils produce oxidizing agents in the form of nitric oxide radicals and reactive oxygen species, the authors speculated that tetrathionate might be produced during inflammation. Indeed, when mice were infected with an $S$. Typhimurium mutant that cannot use tetrathionate, the authors detected tetrathionate in the gut; no tetrathionate was detected after infection with wild-type bacteria, presumably because it was depleted by the bacteria. Inflammation and the production of reactive oxygen species were required for tetrathionate production, as mutant bacteria unable to induce inflammation did not induce tetrathionate production, and no tetrathionate was produced in mice deficient in gp91phox (also known as $\mathrm{CYBB}$ ), an important component in the formation of reactive oxygen species. Competition assays revealed that, in the presence of tetrathionate, wild-type bacteria have a distinct growth advantage over bacteria that cannot use tetrathionate, both in vitro and in an in vivo mouse model. Thus, the induction of inflammation by $S$. Typhimurium results in the production of a compound that gives the bacterium a selective advantage.

Similarly, Bertin and colleagues show that enterohaemorrhagic Escherichia coli (EHEC) can metabolize ethanolamine in the intestines of cows to gain a growth advantage. Ethanolamine is an abundant nutrient derived from the phosphatidylethanolamine in the membranes of intestinal cells that are sloughed off the intestinal wall. EHEC can use this ethanolamine as a nitrogen source. However, the native bacterial population of the cow gastrointestinal tract, which includes other strains of E. coli, does not seem to use ethanolamine efficiently. Competition experiments using wild-type EHEC and mutant EHEC that are unable to use ethanolamine show that ethanolamine utilization provides a distinct growth advantage.

Thus, pathogens can benefit from the exploitation of a nutritional niche in the gastrointestinal tract. This is likely to be a common mechanism by which pathogens gain an edge in colonization of the host.

Christiaan van Ooij

ORIGINAL RESEARCH PAPERS Winter, S. E. et al. Gut inflammation provides a respiratory electron acceptor for Salmonella. Nature $\mathbf{4 6 7}$ 426-429 (2010)|Bertin, Y.et al. Enterohaemorrhagic Escherichia coli gains a competitive advantage by using ethanolamine as a nitrogen source in the bovine intestinal tract. Environ. Microbiol. 16 Sep 2010 (doi: 10.1111/j.1462-2920.2010.02334.x) FURTHER READING Garsin, D. A. Ethanolamine utilization in bacterial pathogens: roles and regulation.. Nature Rev. Microbiol. 8, 290-295 (2010) | Croxen, M. A. \& Finlay, B. B. Molecular mechanisms of Escherichia coli pathogenicity. Nature Rev. Microbiol. 8, 26-38 (2010) 